\title{
The mass transfer process and the growth rate of protein crystals
}

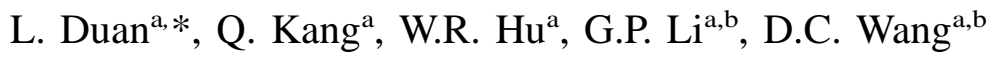 \\ ${ }^{a}$ National Microgravity Laboratory/CAS, Institute of Mechanics, CAS, Beijing 100080, PR China \\ bInstitute of Biophysics, CAS, Beijing 100101, PR China
}

Received 7 February 2002; received in revised form 11 March 2002; accepted 11 March 2002

\begin{abstract}
In this paper, protein crystal growth is studied by a Mach-Zehnder interferometer and an image process system. The interference fringe images are recorded during the crystallization of tetragonal hen egg white lysozyme crystal. Concentration distributions of the protein solution are given from the interference fringe images recorded by the Mach-Zehnder interferometer with a real time servo system of a four-step phase shift. The mass transfer flux and the crystal growth rates are obtained from the concentration distribution. The results show that the observed rates are in accordance with those demonstrated by measurements of the experimental images; therefore the method for determining growth rate by the diffusion process is reasonable. (c) 2002 Elsevier Science B.V. All rights reserved.
\end{abstract}

Keywords: Protein crystal; Crystal growth; Growth rate; Mass transfer; Diffusion; Interferometer

\section{Introduction}

The growth processes of the protein crystal are influenced by many factors, such as impurities, the bunching of growth steps, the formation of defects and the transport process of convection and diffusion. The morphology of the solidification interface is determined mainly by the competition of the transport process and the interface kinetics. The mechanisms dominating the perfection of the protein crystal are still not clear, but modeling experiments of the crystal growth may reveal the details.

The concentration distribution in the solution around the protein crystal is an important feature,

\footnotetext{
*Corresponding author. Tel.: + 86-10-6261-5536; fax: + 8610-6261-5524.

E-mail address: duanli@mail.imech.ac.cn (L. Duan).
}

and is closely related with mass transfer during crystal growth. In recent years, the laser interference method has been used to study the growth of the mineral salt crystal. The growth steps during the process of inorganic crystal growth was observed by using the phase shift optic interference method with high resolution suggested by Onuma and Tsukamoto [1,2], and a similar method was applied to show the concentration transfer by Duan et al. [3]. Further, the coupling action between concentration field and velocity field in the solution was also studied experimentally by Kang et al. [12]. Miyashita et al. studied the growing process of the lysozyme crystal by judging the curve of the interferometer's carrier fringes [4]. Crystal growth rate is one of the key parameters, and has been studied using the atom force micro- 


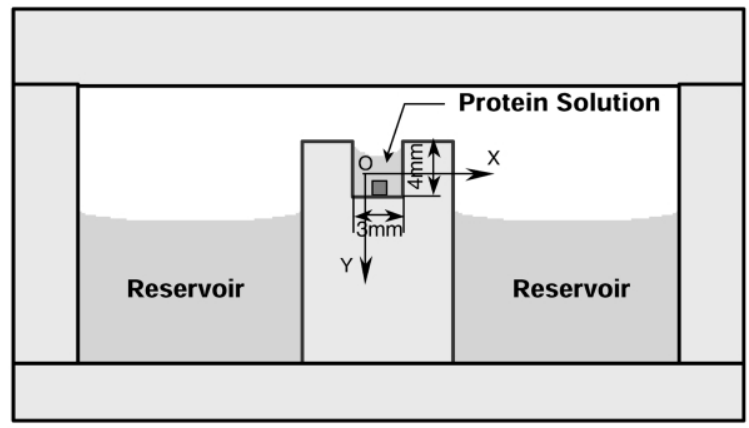

Fig. 1. Schematic diagram of the growth chamber of the protein crystal.

scope (AFM) [5]. Mach-Zehnder interferometer is a promising method to study the process without disturbance. It allows the influences of the concentration field on the growth rate of the protein crystal to be studied in detail by analyzing the pattern of the interference fringes.

The oscillatory pattern during the growth of the protein crystal is crucially important for the perfection of the protein crystal; however, the main focus of the present paper is the discussion of the average properties of the mass transfer process during protein crystal growth. Mass transfer in the solution is demonstrated in the measured interference fringe images recorded by a Mach-Zehnder interferometer using the phase shift method; then the flux of the protein diffusion process is obtained. The results show that the transport process in the solution is mainly dominated by diffusion in comparison with convection, and the results given from the diffusion process are compared with the ones given by direct observation of the crystal growth rates.

\section{Experiment method and the growth rate of protein crystal}

Hen egg white lysozyme from Sigma (Cat, NO L6876, Lot NO 46F-80601), without any further purification before use, was dissolved in doubledeionized water at a concentration of $25 \mathrm{mg} / \mathrm{ml}$, then was centrifuged for $15 \mathrm{~min}$ at $8000 \mathrm{rev} . / \mathrm{min}$. The protein solution was stored in an incubator at $276{ }^{\circ} \mathrm{K}$ until later use. All the other chemicals used in the present experiment were of analytical grade and were used without further purification, but before use, all solutions were subsequently filtered with a micro filter of $0.22 \mu \mathrm{m}$ to reduce noise and ensure success.

Crystallization experiments were conducted by the vapor diffusion method. The facility is suitable for both the vapor diffusion process and optical interference measurement. As shown in Fig. 1, a small sample cell is located in a large reservoir cell, and all the side walls are optical glass. The dimensions of the small sample cell are $3.0 \times 2.0 \times 4.0 \mathrm{~mm}^{3}$. The vapor diffusion crystallization was conducted at a temperature of $292{ }^{\circ} \mathrm{K}$.

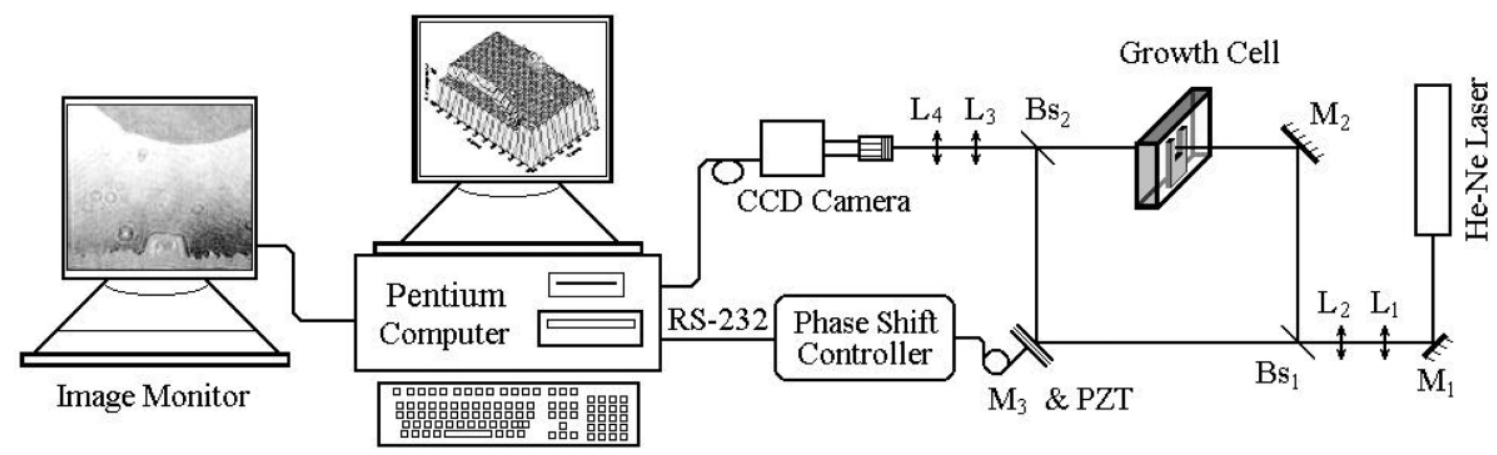

$$
\begin{array}{ll}
\mathrm{Bs}_{1}, \mathrm{Bs}_{2}: \text { Beam Splitter } & \mathrm{L}_{1}, \mathrm{~L}_{2}, \mathrm{~L}_{3}, \mathrm{~L}_{4}: \text { Lens } \\
\mathrm{M}_{1}, \mathrm{M}_{2}, \mathrm{M}_{3} \text { : Mirror } & \mathrm{PZT}: \text { Piezoelectric Translator }
\end{array}
$$

Fig. 2. Schematic diagram of the measurement system. 


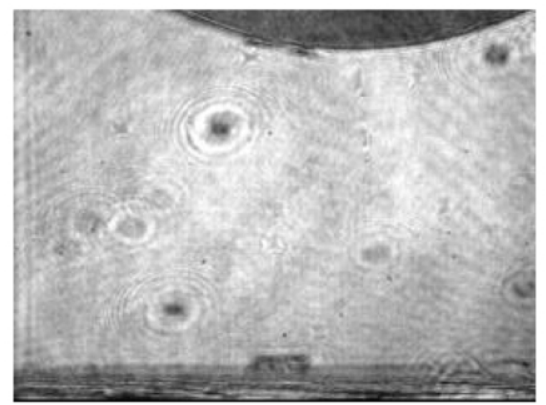

$\mathrm{t}=51.63$ hours

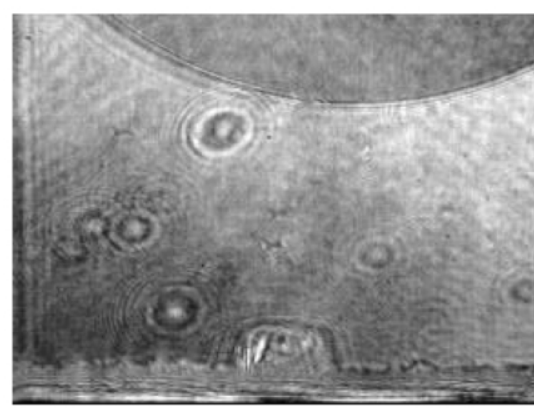

$t=212.23$ hours

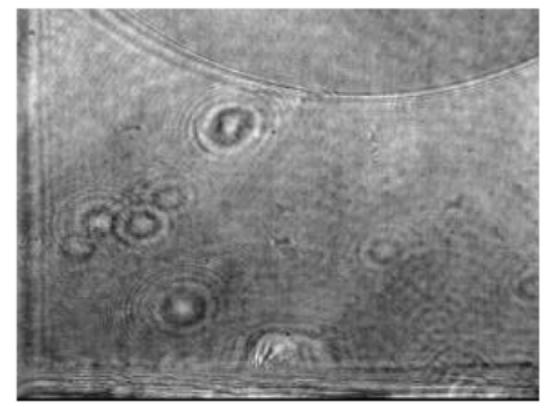

$t=162.12$ hours

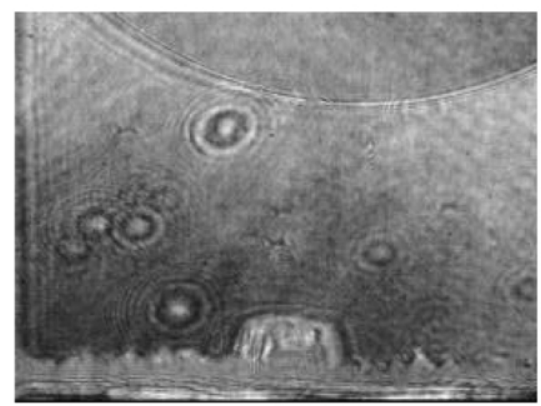

$t=258.23$ hours

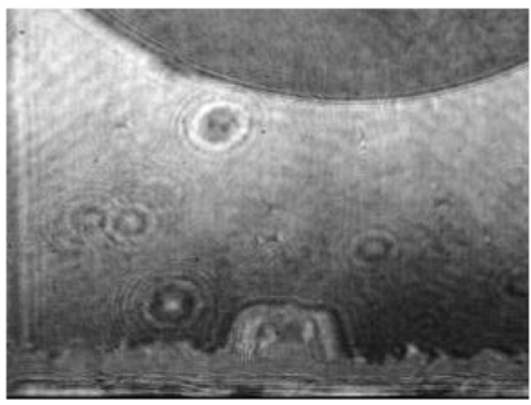

$t=305.18$ hours

Fig. 3. The process of the tetragonal lysozyme crystal growth on the bottom of the cell.

The mother liquor consists of $6 \mu$ l of the lysozyme solution plus $6 \mu \mathrm{l}$ of reservoir solution with $50 \mathrm{mM}$ sodium acetate buffer at $\mathrm{pH}$ 4.5. The mixed mother liquor was injected into the center cell of the chamber. Equilibration was against 400 $\mu l$ reservoir of $1 \mathrm{M} \mathrm{NaCl}$ with the same buffer, which was injected into the large cell of the chamber. The edges of the chamber were smeared with petroleum jelly, and a lid was applied immediately to the top of the chamber to create an airtight seal. Under these conditions, the lysozyme crystallizes in its tetragonal form.

A Mach-Zehnder micro-interferometer with a four-step phase shift system and an image system were used in the experiments as shown in Fig. 2. The field of view of the interferometer was 
$2.0 \times 1.6 \mathrm{~mm}^{2}$. Laser, $\mathrm{L}_{1}, \mathrm{~L}_{2}, \mathrm{M}_{1}, \mathrm{M}_{2}, \mathrm{M}_{3}, \mathrm{Bs}_{1}$ and $\mathrm{Bs}_{2}$ compose a typical Mach-Zehnder interferometer. The mirror $\mathrm{M}_{3}$ was driven by a Piezoelectric Translator (PZT) which was for a phase shift of interferograms. The PZT controller was connected with the computer of the image system. The interferograms were magnified by Lenses L3 and L4, and were recorded by the image system, which was synchronized with the phase shift system of the interferometer. The four-step phase shift technique was used; four images of the interference fringe were acquired to invert fringe images into refractive index field at each stage $[3,6]$.

Crystal growth is very slow, and several days are generally necessary for the experiment period. The fringe images of the micro-interferometer were acquired at a regular interval of one hour, for a total of $1036(=259 \times 4)$ frames of fringe images. A larger crystal setting on the bottom of the growth cell was observed in one of the experiments. The typical sequence of the protein crystal growth is shown in Fig. 3. The shape of the crystal is nearly a cube. The four sides of the crystal are the crystal faces (110), and the top part of the crystal is four crystal faces (101). The crystal has a well-developed regular form except for the bottom crystal faces (101).

The relationship of the refractive index and the concentration of the lysozyme solution was measured by WAY-15 ABBE refractometer in order to convert the refractive index field into a concentration field. The relative formula could be written as follows.

$n=1.349216-0.000227 C$

where, $n$ and $C$ are the refractive index of the protein solution and the concentration of the protein solution, respectively.

Using the technique of the four-step phase shift, the concentration distribution in the solution and the contour maps of the concentration are obtained as shown in Fig. 4. The $X$-axis stands for the length of the measured area, the $Y$-axis stands for the width of the measured area, and the $Z$-axis stands for the transfer of concentration $\Delta C$. The top surface of the protein crystal at the center part of the cell grew from 0 to $0.5 \mathrm{~mm}$ in height during a growth period of $305.18 \mathrm{~h}$.
The image system of the Mach-Zehnder interferometer recorded both interferograms and the shape of the crystal. The positions of faces (101) and faces (110) in crystal growth were measured according to the experimental images of the protein crystal. The evolution of the faces (101) and faces (110) over time is shown in Fig. 5. It clearly shows that the evolution trends of the two curves are parallel even if the sizes of crystals in the two directions are different at the beginning of measurement. The growth rate of the crystal is not uniform throughout the process of crystal growth. It is slow in the first and last stages, and it is nearly uniform in the middle stage. The result of the growth rate is reasonable. In this experiment, the whole observation process of crystal growth by the interferometer is from the time $70 \mathrm{~h}$ to the time $330 \mathrm{~h}$ after configuring the protein crystal growth system, and the total observation period is $260 \mathrm{~h}$. The final size of the crystal cross-section is $0.5 \mathrm{~mm} \times 0.22 \mathrm{~mm}$.

The curve of face (101) in Fig. 5 is expressed by a polynomial of 5 orders as follows:

$$
\begin{aligned}
h= & 4.607 \times 10^{-2}+3.624 \times 10^{-4} t-9.189 \\
& \times 10^{-6} t^{2}+1.312 \times 10^{-7} t^{3}-5.205 \\
& \times 10^{-10} t^{4}+6.417 \times 10^{-13} t^{5}
\end{aligned}
$$

where $h$ is the height of the protein crystal denoted in millimeters; and $t$ is the time of crystal growth denoted in hours. The slopes of the curves in Fig. 5 give the growth rate. According to the Eq. (2), the growth rate of the crystal face (101) could be written as follows:

$$
\begin{aligned}
v_{\text {image }}=\frac{\mathrm{d} h}{\mathrm{~d} t}= & 3.624 \times 10^{-4}-1.838 \times 10^{-5} t \\
& +3.937 \times 10^{-7} t^{2}-2.082 \\
& \times 10^{-9} t^{3}+3.208 \times 10^{-12} t^{4}
\end{aligned}
$$

where $v_{\text {image }}$ stands for the growth rate measured by the images of the crystal, and is denoted in $\mathrm{mm} / \mathrm{h}$. From Eq. (3), the growth rate of face (101) at any time in the process of protein crystal growth can be obtained. In the present work, the growth rates at five different times in the process of crystal growth are given directly by the Eq. (3) to compare with subsequent results of analysis. The results are summarized in Table 1 . The growth rates of face (110) at the same five times are also 

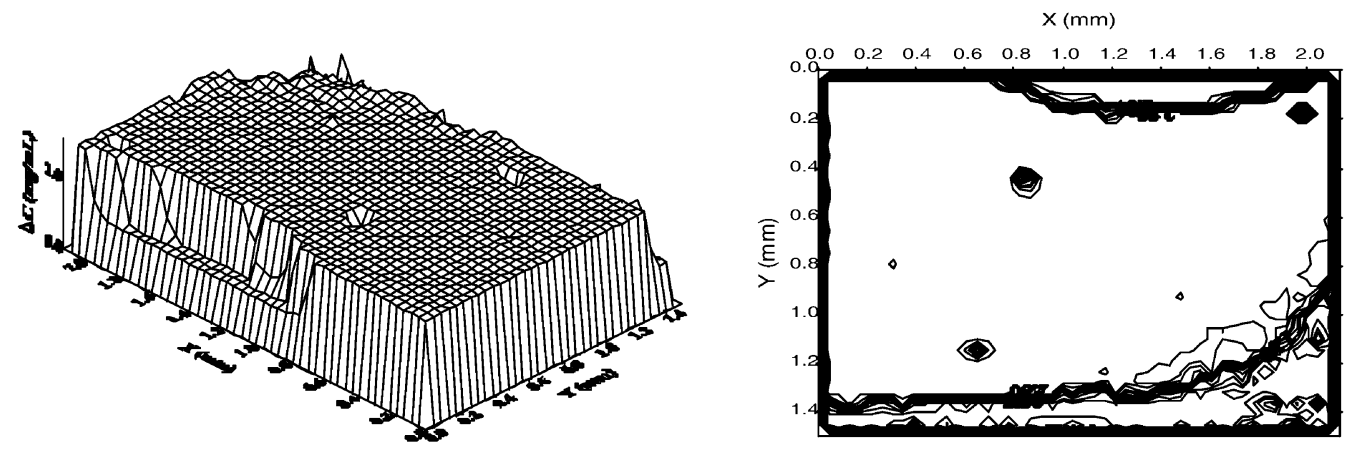

(a) $t=\mathbf{5 1 . 6 3}$ hours
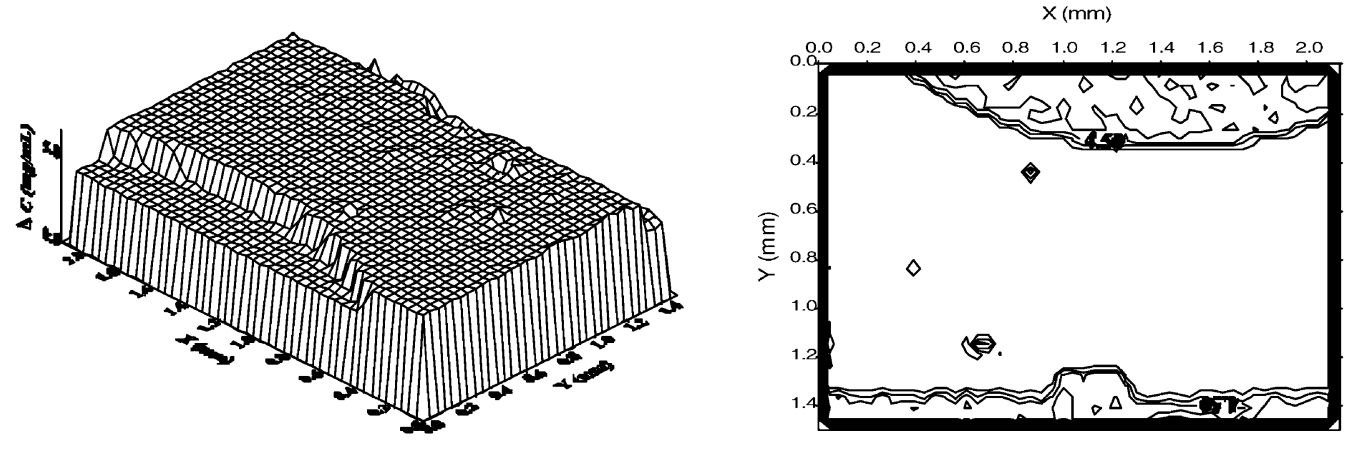

(b) $t=162.12$ hours
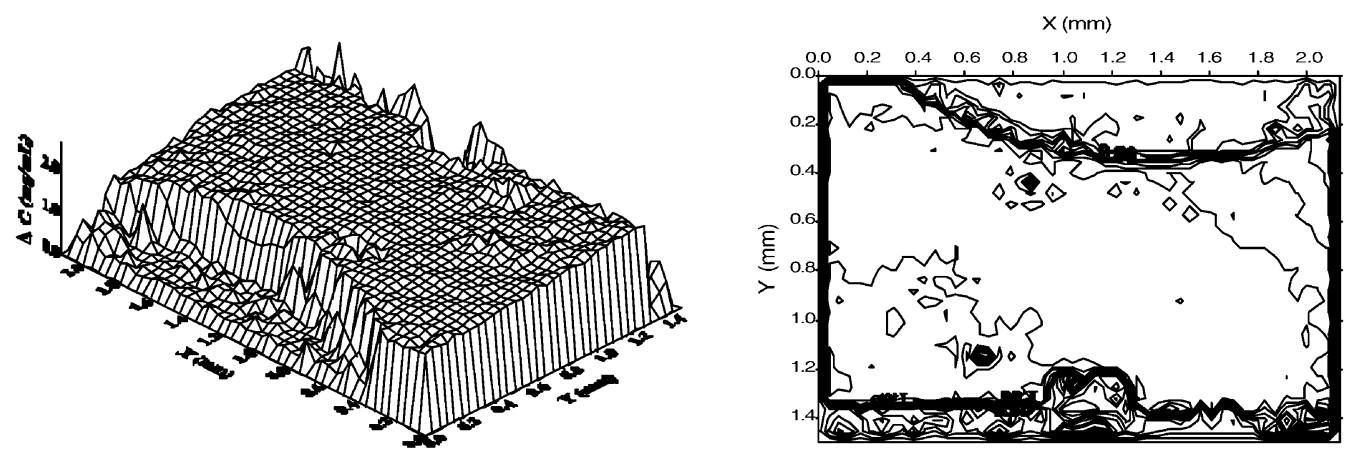

(c) $t=212.23$ hours

Fig. 4. The evolution of the concentration distribution and the contour map. 

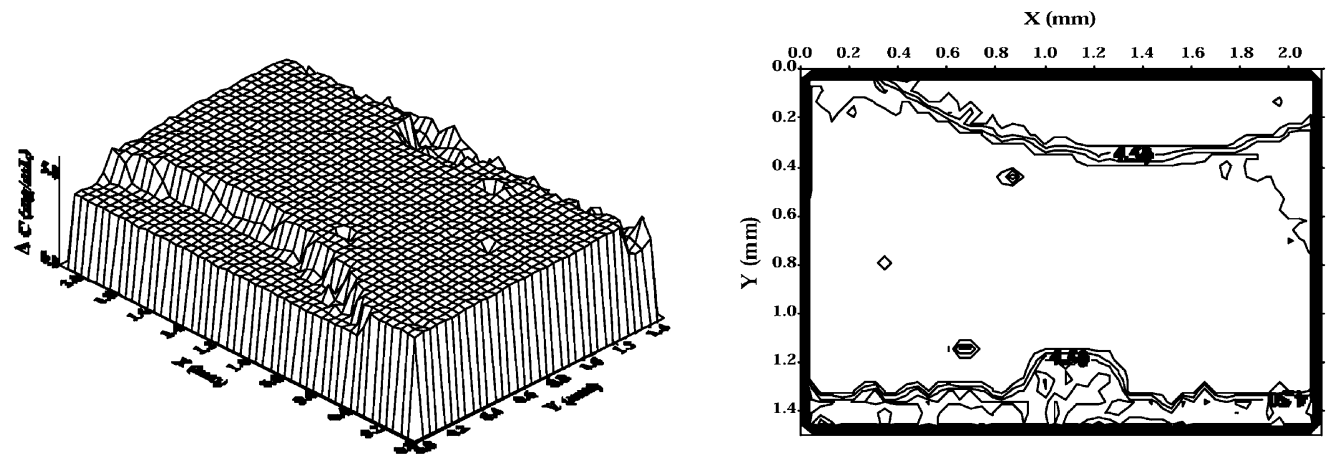

(d) $t=258.23$ hours
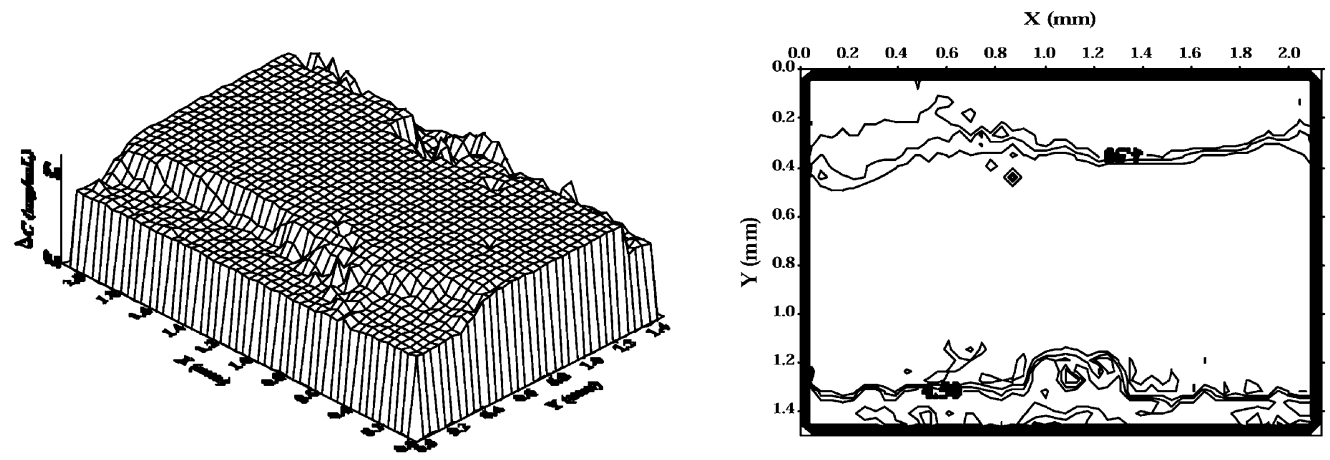

(e) $t=305.18$ hours

Fig. 4 (Continued).

shown in the Table 1 . The growth rate curve that is obtained by directly measuring the images of crystal is shown in Fig. 8c.

\section{The mass transfer process}

The typical scale of the present experiment is only a few millimeters, and the crystallization of the protein crystal is directly correlated with the diffusion process. A constant temperature is maintained through out the experiment. The mass flux $J$ of the protein induced by the diffusion process can be expressed as:

$J=-D \nabla C$

where $D$ is the solute diffusion coefficient, and $\nabla C$ is the gradient of the protein solution concen- tration. The mass of protein in the solution passed through a cross-section face near and parallel to the solidification interface of the crystal will be accumulated mostly on the surface of the crystal. The mass transfer can be approximately calculated by the flux, thus obtaining growth rate. If the growth area of protein crystal is $\Delta S$, the rate of mass transfer $v_{\mathrm{m}}$ can be written as:

$v_{\mathrm{m}}=\frac{\Delta m}{\Delta t}=J \Delta S$

where $\Delta m$ is the protein mass passed through $\Delta S$ in the duration $\Delta t$.

It should be noted that the protein crystals are not only composed of the protein, but also a percentage of solvents. In most cases, the percent- 
age of solvents in the protein crystal may be from 30 to $70 \%$ total weight. The solvent generally occupies large interstices, cavities, and channels within macromolecular crystals, filling them with disordered water and other components. In this paper, the percentage of solvents is considered as $33.5 \%$ of the crystal, as shown in [8]. If all of the proteins passed through area $\Delta S$ are crystallized, the crystal mass will increase $(1+33.5 \%) \Delta m$. So the increase of crystal volume $\Delta V$ can be expressed as:

$\Delta V=\frac{(1+33.5 \%) \Delta m}{\rho}$

where $\rho$ is the density of the crystal. Because the mass transfer is mainly dominated by the diffusion process, if the difference of adsorption on each face of the crystal is considered negligible, the growth rate on every crystal face will be approximately equal since the concentration gradient at each direction of the crystal face is approximately equal under the measurement technique of the experiment. The growth rates of the crystal $v_{\text {flux }}$ could be expressed as follows:

$v_{\text {flux }}=\frac{\Delta h}{\Delta t}=\frac{\Delta V}{\Delta S \Delta t}=\frac{(1+33.5 \%) J}{\rho}$

As long as the concentration distribution is measured by the interferometer, the mass flux and the growth rate can be obtained according to Eq.
Table 1

The growth rate measured from the image of the crystal

\begin{tabular}{llllll}
\hline Time $(\mathrm{h})$ & 51.63 & 162.12 & 212.23 & 258.23 & 305.18 \\
\hline $\begin{array}{c}v_{\text {image }} \text { at face }(101) \\
\left(\mathrm{m} / \mathrm{s} \times 10^{-10}\right)\end{array}$ & 0.596 & 2.986 & 2.224 & 0.786 & 0.196 \\
$\begin{array}{c}v_{\text {image }} \text { at face }(110) \\
\left(\mathrm{m} / \mathrm{s} \times 10^{-10}\right)\end{array}$ & 0.157 & 2.650 & 1.641 & 0.409 & 0.132 \\
\hline
\end{tabular}

(4) and Eq. (7), respectively. The growth rate is directly related to the mass flux $J$ of the protein or to the concentration gradient $\nabla C$ of the protein.

During crystal growth, the growth rates fluctuate along with the concentration distribution. Five typical stages in the process of protein crystal growth will be analyzed now, and the growth rates will be given.

\section{The concentration boundary}

The concentration boundary controls crystal growth and can be observed by the optical diagnostics of this paper. The values of many physical parameters, such as the concentration gradient, the flux, the size of the crystal, the mass depleting rate and the growth rate, etc., are different during the process of crystal growth. The state of the crystal in a typical sample at the growth time $t=$ $212.23 \mathrm{~h}$ will be analyzed here. The growth rate of the crystal at the time would be large, since it

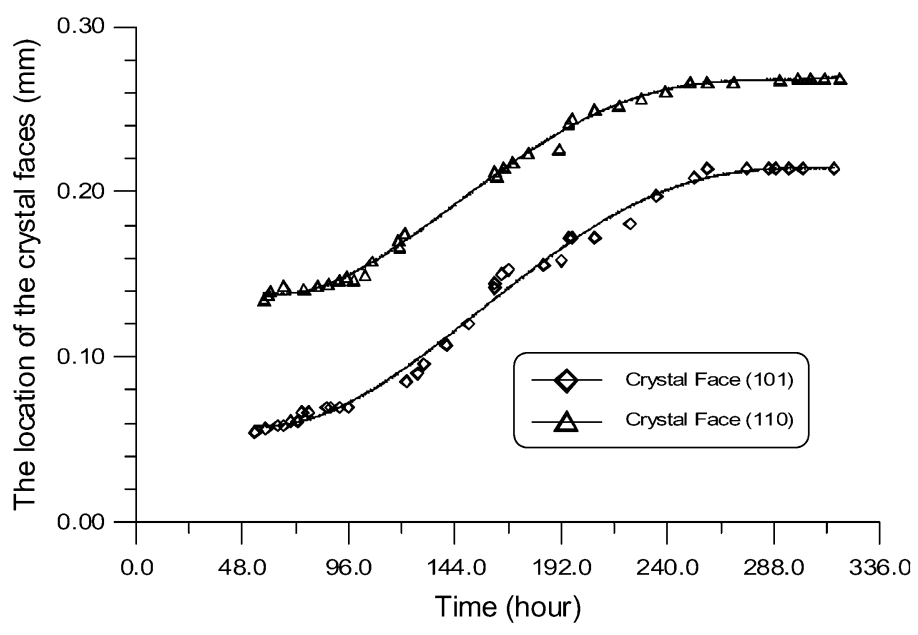

Fig. 5. The evolution of the faces (101) and (110) vs. time. 


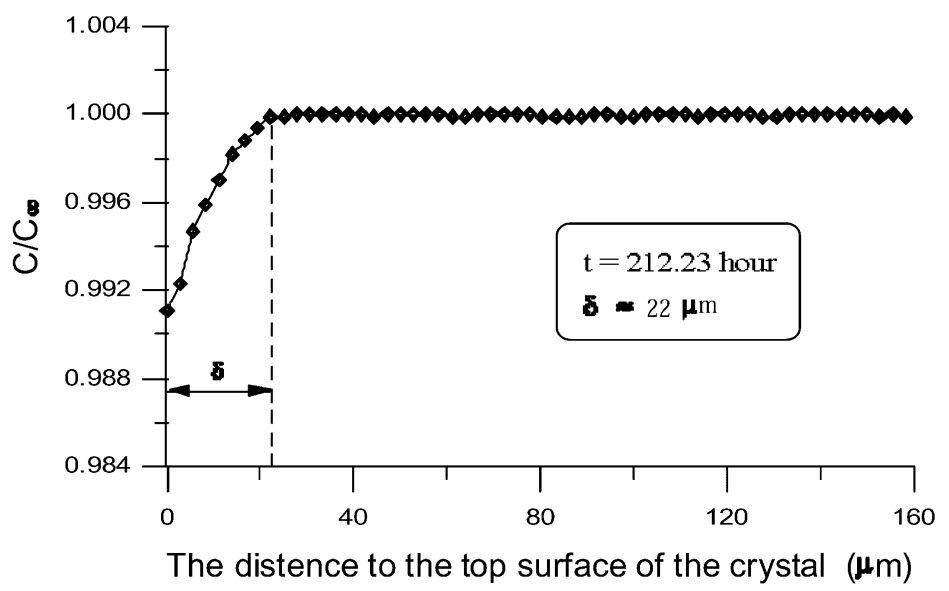

(a) In vertical direction (the center part above crystal)

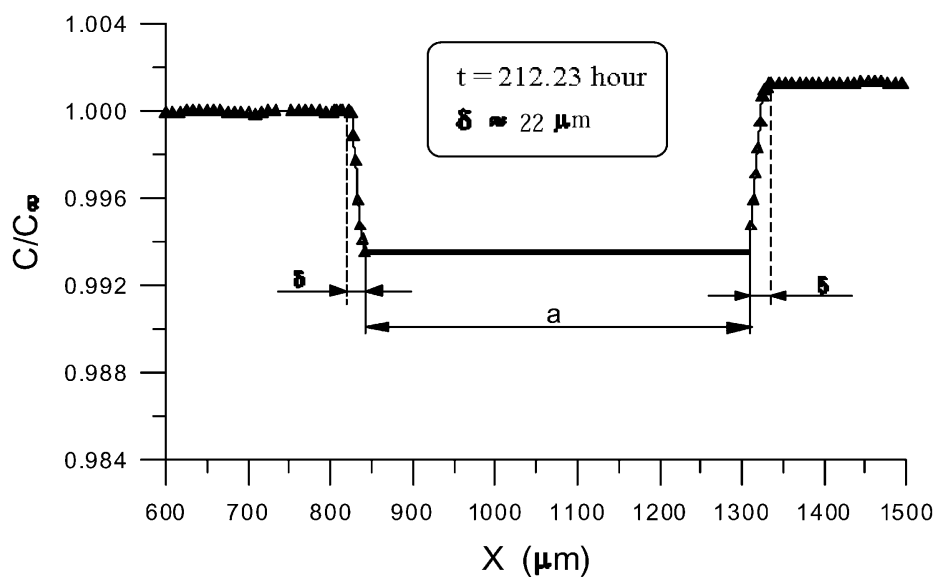

(b) In horizontal direction $(y=1.2842 \mathrm{~mm})$

Fig. 6. The concentration profile at right angles.

is near the middle of the entire process of crystal growth.

The concentration profile above the top crystal face in the vertical direction is given in Fig. 6a. The concentration profile of both side boundaries of the crystal in the horizontal direction (at $Y=$ $1.2842 \mathrm{~mm}$ ) are given in Fig. 6b. In the two figures, $C$ and $C_{\infty}$ are the local solution concen- tration and the solution concentration distant from the crystal, respectively.

The curves in Fig. 6 show the structure of concentration boundary layers and the thickness of the concentration boundary layer. The concentration gradient exists only around the growing protein crystal. The concentration differences of the boundary layer and the thickness of the concentra- 


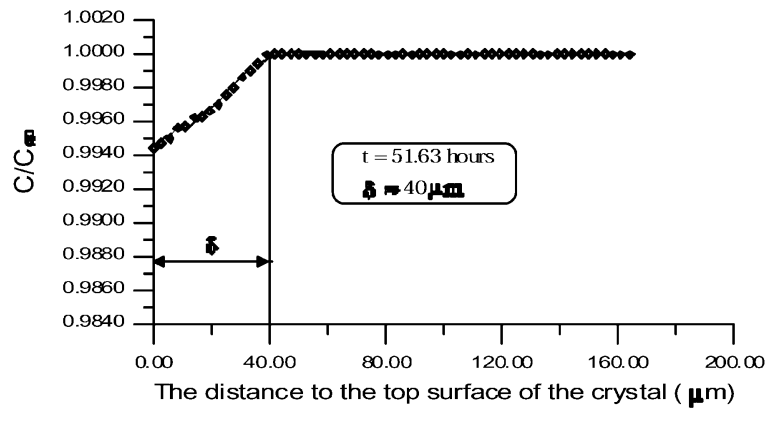

(a)

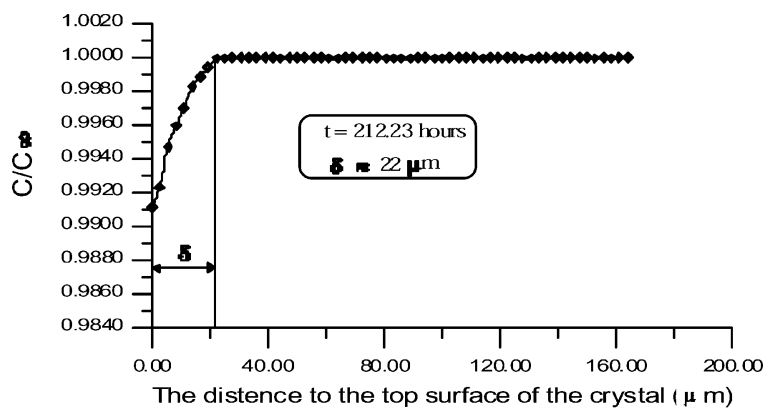

(c)

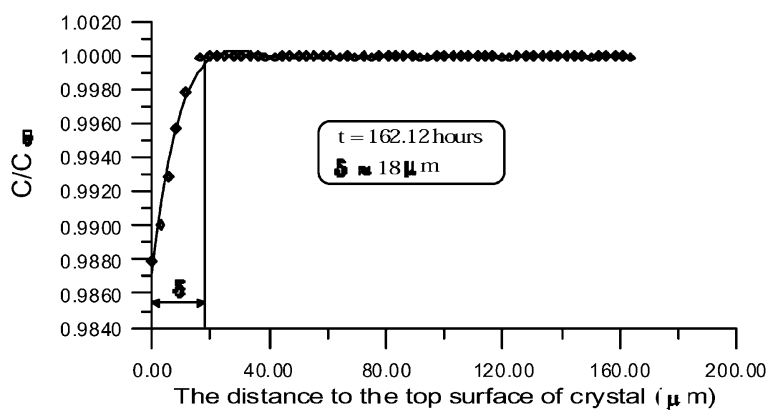

(b)

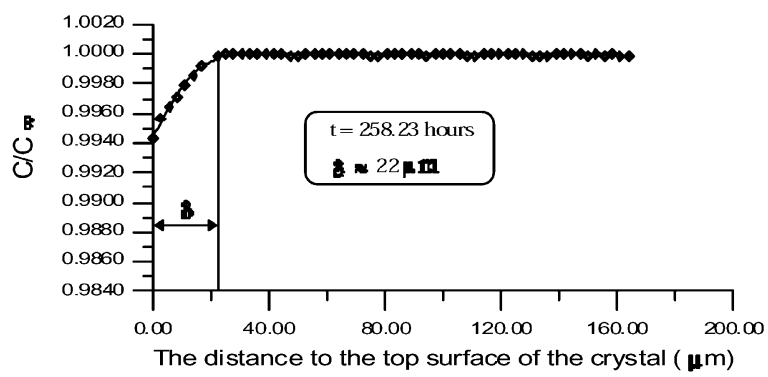

(d)

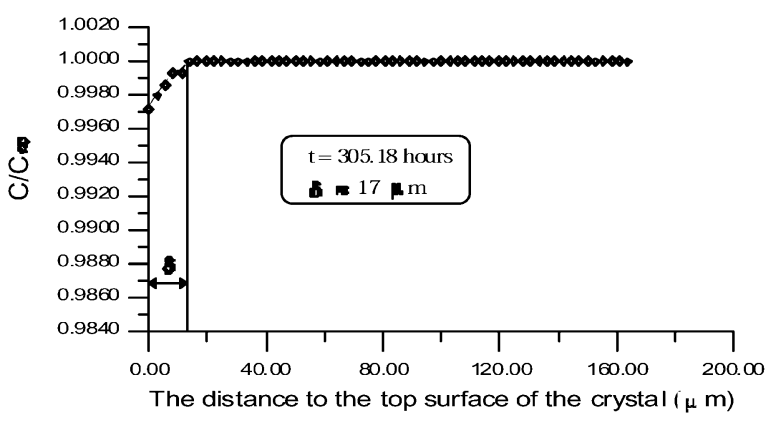

(e)

Fig. 7. The concentration profile at different times.

tion boundary layer are approximately equal at right angles, and the concentration field far from the crystal is uniform. The results show that there may be no buoyancy convection during the process of protein crystal growth. The thickness of the boundary layer $\delta$ and the concentration differences of the boundary layer $\Delta C$ at right angles are 22 $\mu \mathrm{m}$ and $0.2053 \mathrm{mg} / \mathrm{ml}$, respectively, in this case.
The solute diffusion coefficient of the protein system is $D=2 \times 10^{-11} \mathrm{~m}^{2} / \mathrm{s}$ [7]. The flux is:

$$
\begin{aligned}
J & =-2 \times 10^{-11} \times \frac{0.2053}{2.2 \times 10^{-5}} \\
& =-1.866 \times 10^{-7} \mathrm{~kg} /\left(\mathrm{m}^{2} \mathrm{~s}\right)
\end{aligned}
$$

The density of the protein crystal is usually 1.25 $\mathrm{g} / \mathrm{cm}^{3}$. The growth rate calculated by the flux is: 
Table 2

The typical process of the protein crystal growth

\begin{tabular}{cllll}
\hline Time $(\mathrm{h})$ & $\delta(\mu \mathrm{m})$ & $\Delta C(\mathrm{mg} / \mathrm{ml})$ & Flux $J\left(\mathrm{mg} / \mathrm{m}^{2} \mathrm{~s}\right)$ & $v_{\text {flux }}\left(\mathrm{m} / \mathrm{s} \times 10^{-10}\right)$ \\
\hline 53.63 & 40 & 0.1232 & 0.0616 & 0.66 \\
162.12 & 18 & 0.2327 & 0.2585 & 2.76 \\
212.23 & 22 & 0.2053 & 0.1866 & 1.99 \\
258.23 & 22 & 0.1095 & 0.0995 & 1.06 \\
305.18 & 17 & 0.0523 & 0.0615 & 0.66 \\
\hline
\end{tabular}

$$
\begin{aligned}
v_{\text {flux }} & =\frac{(1+33.5 \%) J}{\rho}=\frac{(1+33.5 \%) \times 1.866 \times 10^{-7}}{1.25 \times 10^{3}} \\
& =1.99 \times 10^{-10} \mathrm{~m} / \mathrm{s}
\end{aligned}
$$

The images and data at the other four times, $t=$ $53.63 \mathrm{~h}, 162.12 \mathrm{~h}, 258.23 \mathrm{~h}$ and $305.18 \mathrm{~h}$, are also analyzed. The concentration profile above the top crystal face are given in Fig. 7a-e at these times.

The thickness of the concentration boundary layer, the concentration difference in the boundary layer, the flux and the growth rate are summarized in Table 2 at these times. The curve in concentration difference in the boundary layer changing with time is given in Fig. 8a. The curve in flux changing with time is given in Fig. $8 \mathrm{~b}$. And the curve in growth rate calculated by the flux changing with time is given in Fig. 8c.

In order to compare the calculated growth rate with the measured growth rate, the three curves of growth rate are shown simultaneously in Fig. 8c. The two solid curves in the figure are the growth rates of face (101) and face (110) measured by the images, and the dashed curve stands for the growth rate calculated by flux. From this figure, we find that the evolution trend of the growth rates obtained by the two different methods is almost uniform, and the numerical values of the growth rates $v_{\text {image }}$ and $v_{\text {flux }}$ closely approach each other. These results demonstrate that this method of analysis of protein crystal growth is reasonable, and the mass transfer in the process of protein crystal growth is dominated by the diffusion effect. The diffusion process is the main function in the crystallization of the protein crystal, rather than the buoyancy convection. If the concentration gradient near the crystal face is measured, the growth rate of the crystal can be approximately calculated.
F. Otálora et al. reported that maximum growth rates from $3.6 \mu \mathrm{m} / \mathrm{h}$ to $50 \mu \mathrm{m} / \mathrm{h}$ are observed in SpaceLab1 mission and the Life \& Microgravity Spacelab (Orbiter Mission STS-78) [9,10]. The values of the growth rate are larger than our experimental results. There are several factors which could have caused the difference, such as, microgravity, the size of the lysozyme protein chamber and the size of the salt buffer reservoir, initial protein concentration and salt concentration, growth temperature and growth mechanisms, etc. However, the existence of a maximum growth rate associated with a cross-over between increasing and decreasing growth rates has been clearly observed. And as mentioned in [10], in our experiments the crystal size also shows a linear dependence on the square root of time between two cross-overs related to an initial transient period during nucleation and to the depletion of protein concentration, as shown in Fig. 9. Therefore, the rule of evolution of growth rate of lysozyme crystal is accordant in the two different gravity levels.

\section{Conclusion}

The phase shift Mach-Zehnder method is one of the most promising techniques for demonstrating of the concentration distribution near growing crystals, and is helpful for the study of mass transfer during the crystallization of protein. Most studies pay attention to the morphology of the growth interface and the features of the oscillatory growth rate [11]. In the present paper, the focus is on the mass transfer process near the solidification interfaces in the solution, and the concentration distribution in the cross-section perpendicular to the solidification interfaces are observed in the 


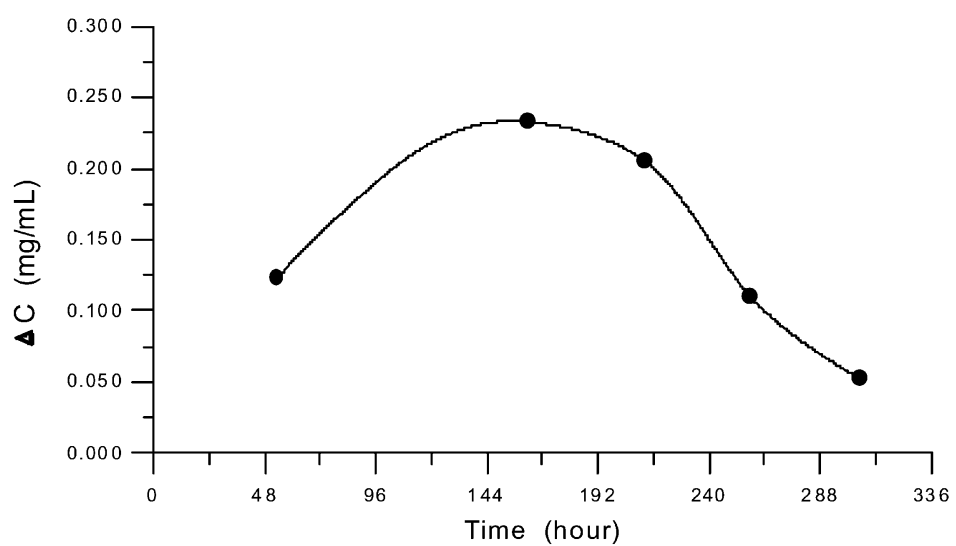

(a)

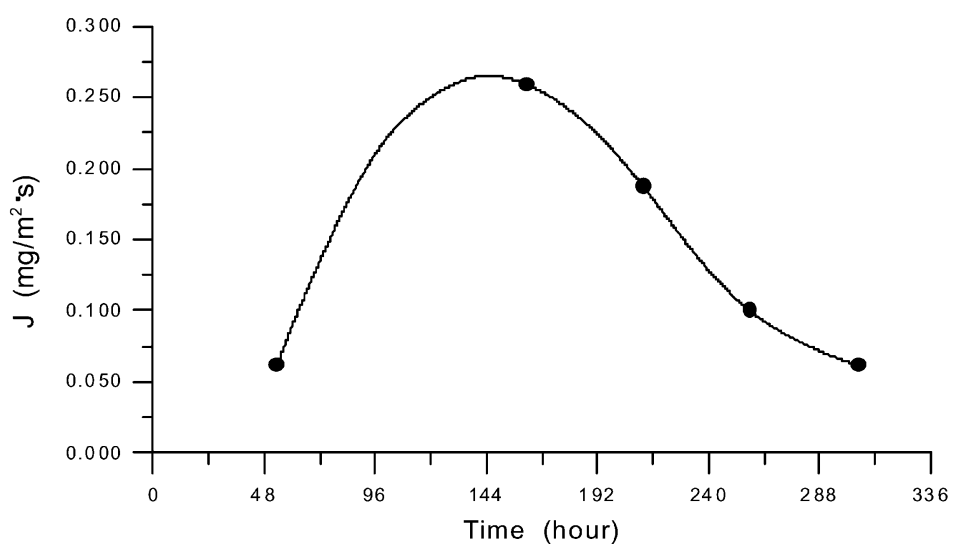

(b)

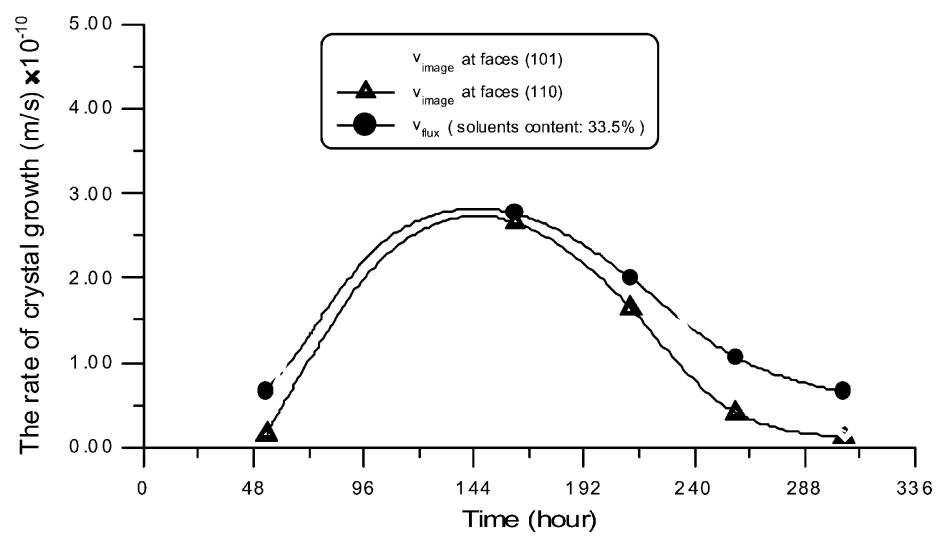

(c)

Fig. 8. Changes in physical parameters vs. time. 


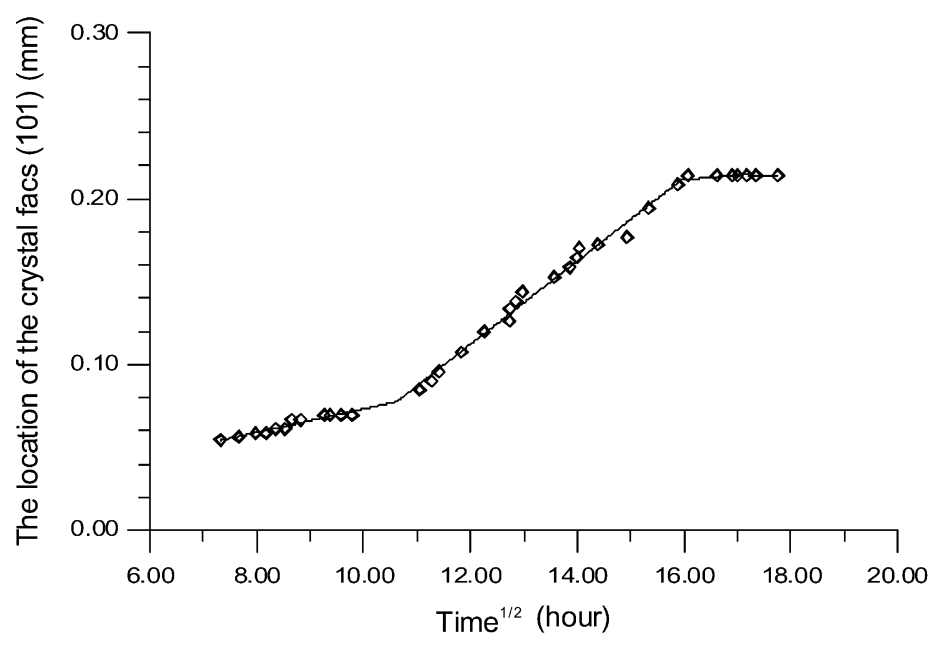

Fig. 9. The evolution of the crystal face (101) vs. the square root of time.

solution. This research may also shed light on the mechanism of protein crystal growth.

The entire process of the protein crystal growth is visualized and measured using a Mach-Zehnder interferometer with a phase-shift servo and an image system. The concentration distributions are obtained by the relationship between the refractive index and the concentration, then evaluating the interference images.

The mass transfer processes are important in the process of protein crystal growth, which were studied in the present experiments. The growth rates of the crystal are obtained by analyzing the concentration gradient and the flux of the mass transfer, and the growth rates are compared with those obtained by directly measuring the size of the crystal. The results show that the analyses of the growth rate are very close to the actual growth of the protein crystal. This kind of analysis method is reasonable, and can predict the growth rate of the protein crystal by studying the concentration distribution of the solution near the growing crystal. The mass transfer during the entire process of crystallization of the protein is mainly dominated by the diffusion in comparison with the convection. The trend of the growth rate of the lysozyme crystal is in agreement with the results of groundbased experiments and the space experiments in the microgravity environment.

\section{Acknowledgments}

The work was supported by the Grant 95 -yu-34 of the Ministry of Science and Technology of China, and by the Foundation of China Post Doctor.

\section{References}

[1] K. Onuma, K. Tsukamoto, I. Sunagawa, Role of buoyancy driven convection in aqueous solution growth; a case study of $\left(\mathrm{BaNO}_{3}\right)_{2}$ crystal, J. Cryst. Growth 89 (1988) 177-188.

[2] K. Onuma, T. Kameyama, K. Tsukamoto, In situ study of surface phenomena by real time phase shift interferometry, J. Cryst. Growth 137 (1994) 610-622.

[3] L. Duan, J.Z. Shu, The convection during $\mathrm{NaClO}_{3}$ crystal growth observed by real time phase shift interferometry, J. Cryst. Growth 223 (2001) 181-188.

[4] S. Miyashita, H. Komatsu, Y. Suzuki, et al., Observation of the concentration distribution around a growing lysozyme crystal, J. Cryst. Growth 141 (1994) 419-424.

[5] S.D. Durbin, W.E. Carlson, Lysozyme crystal growth studied by atomic force microscopy, J. Cryst. Growth 122 (1992) 71-79.

[6] J. Schmit, et al., Extended averaging technique for deviation of error-compensating algorithms in phaseshifting interferometry, Appl. Opt. 34 (19) (1995) 3610-3619.

[7] W. Eberstein, Y. Georgalis, W. Saenger, Molecular interactions in crystallizing lysozyme solutions studied by photon correlation spectroscopy, J. Cryst. Growth 143 (1994) 71-78. 
[8] L.K. Steinrauf, Preliminary X-ray data for some new crystalline forms of $\beta$-lactoglobulin and hen-egg-white lysozyme, Acta Cryst. 12 (1959) 77-79.

[9] J.M. Garcia-Ruiz, F. Otáalora, Crystal growth studies in microgravity with the APCF (II. Image analysis studies), J. Cryst. Growth 182 (1997) 155-167.

[10] F. Otáalora, M. Lusa Novella, et al., Growth of lysozyme crystals under microgravity conditions in the LMS(STS78) mission, J. Cryst. Growth 196 (1999) 649-664.
[11] P.G. Vekilov, J.W.D. Alexander, F. Ronsenberger, Nonlinear response of layer growth dynamics in the mixed kinetics-bulk-transport regime, Phys. Rev. E 54 (1996) 6650.

[12] Q. Kang, L. Duan, W.R. Hu, Mass transfer process during the $\mathrm{NaClO}_{3}$ crystal growth process, Int. J. Heat Mass Transfer 44 (2001) 3213-3222. 